\title{
Effect of unilateral subthalamic deep brain stimulation in highly asymmetrical Parkinson's disease: 7-year follow-up
}

\author{
Gwanhee Ehm, MD, ${ }^{1}$ Han-Joon Kim, MD, PhD, ${ }^{2}$ Ji-Young Kim, MD, PhD, ${ }^{3}$ Jee-Young Lee, MD, PhD, ${ }^{4}$ \\ Hee Jin Kim, MD, ${ }^{5}$ Ji Young Yun, MD, PhD, ${ }^{6}$ Young Eun Kim, MD, ${ }^{7}$ Hui-Jun Yang, MD, ${ }^{8}$ \\ Yong Hoon Lim, BS, ${ }^{9}$ Beomseok Jeon, MD, PhD, ${ }^{2}$ and Sun Ha Paek, MD, PhD ${ }^{9}$
}

\begin{abstract}
${ }^{1}$ Department of Neurology, National Medical Center; ${ }^{2}$ Department of Neurology and Movement Disorder Center, Parkinson Study Group, and Neuroscience Research Institute, College of Medicine, Seoul National University; ${ }^{3}$ Department of Neurology, Inje University Seoul Paik Hospital; ${ }^{4}$ Department of Neurology, College of Medicine, Seoul National University, Metropolitan Boramae Hospital; ${ }^{5}$ Department of Neurology, Konkuk University Medical Center; ${ }^{6}$ Department of Neurology, Ewha Women's University College of Medicine; ' ${ }^{2}$ Department of Neurosurgery, Seoul National University Hospital, College of Medicine, Seoul National University, Seoul; ' ${ }^{2}$ epartment of Neurology, Hallym University Sacred Heart Hospital, Hallym University College of Medicine, Anyang-si; and ${ }^{8}$ Department of Neurology, Ulsan University Hospital, University of Ulsan College of Medicine, Ulsan, Korea
\end{abstract}

OBJECTIVE For patients with highly asymmetrical Parkinson's disease (PD), unilateral subthalamic nucleus (STN) deep brain stimulation (DBS) has been suggested as a reasonable treatment. However, the results of a previous 2-year followup study involving patients with prominently asymmetrical PD who had unilateral STN DBS suggested that simultaneous bilateral surgery should be performed. In the present study, the authors analyze 7-year follow-up data from the same patient group to examine changes in motor benefit from unilateral STN DBS over time and the interval between initial unilateral surgery and a second (contralateral) STN DBS surgery.

METHODS Eight patients with highly asymmetrical parkinsonism who underwent unilateral STN DBS were evaluated. The factors measured were scores on the motor part of the Unified Parkinson's Disease Rating Scale (UPDRS III), Hoehn and Yahr (HY) stage, and levodopa equivalent daily dose (LEDD). Evaluations occurred at 3, 6, and 12 months after the initial surgery and annually thereafter.

RESULTS The mean follow-up period was 91.5 months (range 36-105 months). Three years after the initial unilateral surgery, motor benefits on the contralateral side continued; however, an aggravation of the ipsilateral parkinsonism attenuated the improvement in total UPDRS III scores, which reverted to baseline. Axial motor score, LEDD, and HY stage did not differ from the baseline. Seven of 8 patients (87.5\%) were considered candidates for a second surgery to offer additional motor benefits. Of the 7 candidates, 4 patients (50\% of total patients) underwent the second surgery at $58.5 \pm$ 11.6 (mean \pm SD) months after the initial surgery. Three patients were not able to have the second surgery: one patient died of gastric cancer, one patient was severely immobilized by an accident, and one patient could not afford the second surgery. One patient remained content with the initial unilateral surgery throughout the follow-up period.

CONCLUSIONS Seven of 8 patients with unilateral STN DBS became candidates for second surgery before battery replacement surgery of the first implanted device. Baseline asymmetry alone may not predict appropriate candidates for unilateral STN DBS. This study provides further evidence that, from a long-term perspective, initial simultaneous bilateral STN DBS should be considered for PD patients with prominently asymmetrical motor symptoms.

https://thejns.org/doi/abs/10.3171/2018.5.JNS172006

KEYWORDS Parkinson's disease; unilateral deep brain stimulation; asymmetry; functional neurosurgery

$\mathrm{B}$ ILATERAL electrode implantation is regarded as the standard procedure for subthalamic nucleus (STN) deep brain stimulation (DBS) for patients with advanced Parkinson's disease (PD). ${ }^{1}$ This is based on the fact that PD affects both sides of the body and bilateral sur- gery provides greater motor benefits than unilateral surgery. ${ }^{2,6,11}$ Under certain conditions, such as patients with prominently asymmetrical motor symptoms, several studies have suggested that unilateral surgery is a reasonable treatment option because it can provide significant bilater-

ABBREVIATIONS DBS = deep brain stimulation; HY = Hoehn and Yahr; LEDD = levodopa equivalent daily dose; PD = Parkinson's disease; PIGD = postural instability/gait disturbance; STN = subthalamic nucleus; UPDRS III = Unified Parkinson's Disease Rating Scale Part III.

SUBMITTED August 14, 2017. ACCEPTED May 29, 2018.

INCLUDE WHEN CITING Published online November 23, 2018; DOI: 10.3171/2018.5.JNS172006. 
al and axial improvements with a lower risk of complications and lower cost. ${ }^{13-15}$ However, PD symptoms become progressively worse on both sides and the axial part of the body; thus, it is expected that even highly asymmetrical PD patients will eventually become unsatisfied with the single-electrode stimulation. Therefore, the usefulness of unilateral surgery depends on the duration over which it can provide adequate benefits for patients until a second surgery becomes necessary.

We have previously reported a 2-year follow-up study with 8 highly asymmetrical patients who underwent unilateral surgery. ${ }^{4}$ The results of the previous study suggested that simultaneous bilateral STN DBS should be considered even in highly asymmetrical patients. ${ }^{4}$ However, this conclusion could be challenged because none of the patients had undergone a second surgery during the study period. The current study aimed to define the period for which highly asymmetrical PD patients are content with unilateral STN DBS alone. For this purpose, the same patients were further followed to examine serial changes in motor benefits from unilateral surgery, the incidence of second surgery, and the interval between first and second surgery.

\section{Methods \\ Patients}

All patients, surgical procedures, and evaluations are as described in our previous study. ${ }^{4}$ Briefly, we included 8 patients from a total of 57 who underwent STN DBS surgery between May 2005 and April 2006 at the Movement Disorder Center of Seoul National University Hospital. All 8 patients had highly asymmetrical parkinsonism, which was defined as when the motor Unified Parkinson's Disease Rating Scale (UPDRS III) score of the more affected side was at least twice the score of the less affected side. We performed follow-up evaluations on the 8 patients up to 7 years after the first unilateral surgery (mean follow-up time of 91 months). The study protocol was approved by the Seoul National University Hospital Institutional Review Board and conformed to the principles of the Declaration of Helsinki. Patient consent was not required due to the retrospective nature of the study.

\section{Evaluation}

The pre- and postoperative evaluation protocols generally followed the CAPSIT-PD guidelines. UPDRS and Hoehn and Yahr (HY) stages were assessed in both on- and off-medication states, and postoperative evaluation included additional on- and off-stimulation states. The ipsilateral or contralateral motor UPDRS subscores referred to the sum of the scores for limb tremor, limb rigidity, and limb akinesia (items 20-26), and the axial motor UPDRS subscore was the sum of the scores for speech, facial expression, chin-tongue tremor, neck rigidity, rising from chair, posture, gait, postural instability, and body bradykinesia (items 18-20, 22 for neck, and 27-31). The levodopa equivalent daily dose (LEDD) was calculated as follows: $100 \mathrm{mg}$ of standard levodopa $=140 \mathrm{mg}$ of controlled-release levodopa $=1 \mathrm{mg}$ of pramipexole $=4$ $\mathrm{mg}$ of ropinirole. Postoperative evaluations after the first
TABLE 1. Demographic characteristics, disease duration, and DBS targets

\begin{tabular}{ccccc}
\hline Patient No. & Age $(\mathrm{yrs})^{*}$ & Sex & Disease Duration (yrs)† & Target \\
\hline 1 & 56 & $\mathrm{~F}$ & 8 & Lt STN \\
\hline 2 & 47 & $\mathrm{M}$ & 4 & Lt STN \\
\hline 3 & 52 & $\mathrm{M}$ & 7 & Lt STN \\
\hline 4 & 72 & $\mathrm{M}$ & 5 & Lt STN \\
\hline 5 & 45 & $\mathrm{M}$ & 3 & Rt STN \\
\hline 6 & 63 & $\mathrm{~F}$ & 10 & Lt STN \\
\hline 7 & 53 & $\mathrm{~F}$ & 6 & Rt STN \\
\hline 8 & 69 & $\mathrm{M}$ & 11 & Rt STN \\
\hline
\end{tabular}

* Mean $57.1 \pm 9.9$ years.

† Mean $6.8 \pm 2.8$ years.

unilateral surgery were performed at 3,6, and 12 months after the initial surgery. Following this, evaluations were carried out every 12 months.

\section{Statistics}

Changes in motor UPDRS subscores, HY stage, and LEDD were analyzed using paired t-tests based on the assumption of a normal distribution. Statistical significance was set at $\mathrm{p}<0.05$. All data were analyzed using Stata (Stata Statistical Software, Release 10; StataCorp LLC).

\section{Results}

The demographic characteristics of the 8 patients as well as the duration of PD and the DBS target used in the initial surgery are shown in Table 1. During the followup period, 1 patient died of gastric cancer at 36 months after the first surgery (patient 4). When this patient was included in the statistics, the mean total follow-up period from the first unilateral surgery to the last follow-up was 91.5 months (range 36-105 months). Seven of the 8 patients were considered candidates for a second surgery as the effects of unilateral STN stimulation and medication gradually became unsatisfactory. The patients complained of symptom disparity between the 2 sides of the body because one side was controlled by electrode stimulation, and the other, unstimulated side was treated with medication alone. The optimal therapeutic window of a "good" on-medication state, which is between the off-medication state and dyskinesia, narrows as PD progresses. In these circumstances, the medication dose was elevated to alleviate motor symptoms of the unstimulated side; however, this induced a disabling peak-dose dyskinesia on the stimulated side. Conversely, when the medication dose was reduced to control peak-dose dyskinesia, the unstimulated side entered the off-medication state. Despite meticulous adjustment of simultaneous electrode stimulation and medication dosages by a single experienced neurologist (B.J.), this situation became increasingly difficult to manage over time. Eventually, 4 of 8 patients $(50 \%)$ underwent a second surgery at a mean $( \pm \mathrm{SD})$ of $58.5 \pm 11.6$ months after the first surgery. Three other patients were candidates but were not able to undergo the second surgery (patient 4 died of gastric cancer at 36 months after the first surgery; 
TABLE 2. Baseline and follow-up clinical characteristics

\begin{tabular}{|c|c|c|c|c|c|c|c|}
\hline \multirow{2}{*}{$\begin{array}{l}\text { Patient } \\
\text { No. }\end{array}$} & \multicolumn{3}{|c|}{ Baseline UPDRS III Scores* } & \multirow{2}{*}{$\begin{array}{l}\text { Interval Btwn 1st } \\
\& \text { 2nd Ops (mos) }\end{array}$} & \multirow{2}{*}{$\begin{array}{l}\text { Total Follow-Up } \\
\text { Period (mos)§ }\end{array}$} & \multirow{2}{*}{$\begin{array}{l}\text { Candidate } \\
\text { for 2nd Op }\end{array}$} & \multirow{2}{*}{$\begin{array}{l}\text { Reason 2nd Op } \\
\text { Not Performed }\end{array}$} \\
\hline & Total† & Right & Left & & & & \\
\hline 1 & 37 & 21.5 & 9.5 & 72 & 105 & Yes & NA \\
\hline 2 & 22.5 & 17 & 1.5 & 63 & 104 & Yes & NA \\
\hline 3 & 24 & 13 & 6.5 & 45 & 104 & Yes & NA \\
\hline 4 & 37 & 19 & 7 & NA & 36 & Yes & Death \\
\hline 5 & 50.5 & 25 & 12 & 54 & 99 & Yes & NA \\
\hline 6 & 35 & 17.5 & 6.5 & NA & 88 & Yes & Femur fracture \\
\hline 7 & 34.5 & 16.5 & 8 & NA & 100 & Yes & Financial issue \\
\hline 8 & 26.5 & 15 & 7 & NA & 96 & No & NA \\
\hline \multicolumn{8}{|c|}{$\begin{array}{l}\text { NA = not applicable. } \\
{ }^{*} \text { Off medication. } \\
\dagger \text { Mean } 33.4 \pm 9.1 . \\
\ddagger \text { Mean } 58.5 \pm 11.6 . \\
\text { § Mean } 91.5 \pm 23.1\end{array}$} \\
\hline
\end{tabular}

patient 6 was severely immobilized by an accident at 43 months; and patient 7 could not afford the additional surgery). Patient 8 was satisfied with the unilateral STN DBS throughout the follow-up period (Table 2).

Table 3 and Fig. 1 present data from all patients during the 3-year postoperative follow-up period after the initial surgery. As shown in Table 3 , the off-medication total UPDRS III score improved by $42 \%$ and $31 \%$ at 1 and 2 years' follow-up, respectively; however, at the 3 -year follow-up, the mean total UPDRS III score returned to baseline. The mean UPDRS III subscore in the contralateral side was reduced by $68 \%, 72 \%$, and $60 \%$ at 1,2 , and 3 years after surgery, respectively. There was no change in the mean axial UPDRS III subscore up to 3 years after surgery. The mean ipsilateral UPDRS III subscore worsened by $41 \%$ and $98 \%$ at 2 and 3 years after surgery, respectively. The mean LEDD decreased by $39 \%$ at 1 year after surgery, but there was no significant change at 2 and 3 years. In summary, the mean total UPDRS III score, axial UPDRS III subscore, HY stage, and LEDD remained at or returned to baseline at 3 years after the initial surgery.

The improvement in the contralateral UPDRS III sub- score persisted for 3 years. The ipsilateral UPDRS III subscore worsened gradually and doubled at 3 years after the initial surgery. The direction of asymmetry of motor symptoms, measured by UPDRS, reversed between 3 and 6 months after the first surgery (Fig. 1). Figure 2 shows the UPDRS III scores of the 4 patients who had the second surgery up to their last follow-up visit (mean 103 and 45 months after the first and second surgery, respectively). After the second surgery, all 4 patients experienced additional improvements in the relevant side.

Systematic analysis of clinical parameters in all 8 patients after 3 years after the initial surgery was not possible. Data from patients 4 and 6 were not available (patient 4 died at 36 months after the initial surgery and patient 6 was immobilized by an accident at 43 months after the initial surgery, and thus meaningful UPDRS scores could not be evaluated). While patients 7 and 8 were evaluated later than 3 years after the initial surgery, patients 2, 3, and 5 underwent the second surgery at different time points; therefore, mean UPDRS III subscores of all 8 patients could not be determined later than 3 years after the first surgery.

TABLE 3. Clinical outcomes of unilateral STN DBS for the first 3 years after surgery

\begin{tabular}{|c|c|c|c|c|c|c|c|}
\hline \multirow[b]{2}{*}{ Variable } & \multirow[b]{2}{*}{ Baseline* } & \multicolumn{3}{|c|}{ Follow-Up After 1st Op* } & \multicolumn{3}{|c|}{$\mathrm{p}$ Value (vs baseline) $\dagger$} \\
\hline & & $1 \mathrm{Yr}$ & 2 Yrs & 3 Yrs & $1 \mathrm{Yr}$ & 2 Yrs & 3 Yrs \\
\hline \multicolumn{8}{|l|}{ UPDRS III } \\
\hline Total & $33.4 \pm 9.1$ & $19.5 \pm 11.4$ & $22.9 \pm 11.3$ & $32.3 \pm 14.1$ & 0.010 & $<0.001$ & 0.769 \\
\hline Contralateral $\ddagger$ & $18.1 \pm 3.8$ & $5.9 \pm 4.6$ & $5.1 \pm 4.5$ & $7.3 \pm 4.0$ & $<0.001$ & $<0.001$ & $<0.001$ \\
\hline Axial & $8.1 \pm 3.7$ & $6.1 \pm 3.6$ & $7.6 \pm 3.9$ & $10.4 \pm 5.7$ & 0.142 & 0.624 & 0.141 \\
\hline Ipsilateralł & $7.3 \pm 3.0$ & $7.6 \pm 4.2$ & $10.3 \pm 4.2$ & $14.5 \pm 6.1$ & 0.883 & 0.034 & 0.010 \\
\hline HY & $2.3 \pm 0.5$ & $2.1 \pm 0.6$ & $2.2 \pm 0.7$ & $2.8 \pm 1.0$ & 0.285 & 0.516 & 0.133 \\
\hline LEDD & $676.3 \pm 216.8$ & $409.8 \pm 208.0$ & $567.4 \pm 319.8$ & $671.9 \pm 260.7$ & 0.021 & 0.423 & 0.972 \\
\hline
\end{tabular}

Data are mean values $( \pm S D)$ for the group of 8 patients.

* Off medication. UPDRS III scores were obtained in the off-medication and on-stimulation state.

$\uparrow$ Based on paired t-tests.

$\ddagger$ Relative to the implanted electrodes. 


\section{Discussion}

The current study demonstrates 2 principal findings. First, the severity of the motor symptoms of patients with highly asymmetrical PD returned to baseline levels, with a side-reversed pattern, 3 years after the initial unilateral STN DBS. Second, $50 \%$ of patients with highly asymmetrical motor symptoms underwent a second surgery at 5 years after the initial surgery. Our previous 2-year followup study had suggested that the beneficial period of satisfactory symptom relief from unilateral STN DBS should be considered when selecting candidates for this surgery. In addition, bilateral surgery is more appropriate even in patients with highly asymmetrical PD. ${ }^{4}$ However, the mean total UPDRS III score at 2 years after a first unilateral surgery was lower than the baseline score (23 vs 33 ), and no patients underwent the second surgery during the initial 2-year follow-up period. Our suggestion in the previous study ${ }^{4}$ was based on the clinicians' anticipation that symptom relief from unilateral stimulation would eventually be insufficient, and on the patients' hopes that the second surgery would further improve motor symptoms. Thus, we sought clinical evidence to support our suggestion in the previous 2-year follow-up study and to clarify the interval between the first and second surgery.

In this study, we assessed the same patients as previously for a mean follow-up period of 7 years. We found that there was an improvement in symptoms on the contralateral side after the first unilateral surgery and that the improvement persisted for 3 years. However, significant relief from symptoms on the ipsilateral side only persisted for the first 6 months. Furthermore, the ipsilateral UPDRS III score began to deteriorate at 2 years after the initial surgery. There was no significant change in axial UPDRS III scores over 3 years. Taken together, our results showed that in the off-medication and on-stimulation state, the severity pattern of motor symptoms became reversed (i.e., the milder side became the more severe side), with axial and total UPDRS III scores equivalent to the baseline scores at 3 years after the initial surgery.

We offered thorough discussions regarding the second surgery to all candidates and caregivers. A major consideration was the timing of the second surgery, because all candidates were going to require battery replacement surgery. The battery longevity of the DBS device (Soletra; Medtronic, Inc.) is approximately 5 years in STN DBS for PD. ${ }^{10}$ Discussions regarding a second surgery occurred in advance of the scheduled battery replacement of the initially implanted device. Therefore, if patients underwent the second surgery prior to battery replacement, they would require separate battery replacement operations for the two sides in the future. Alternatively, despite unsatisfactory symptom control, the second surgery could be delayed until the scheduled battery replacement surgery to reduce the number of operations. After careful consideration, all 4 candidates and caregivers agreed to undergo the second DBS unit placement simultaneously with the upcoming battery replacement procedure. Thus, the interval from first to second surgery was approximately 5 years (mean 59 months, range 45-72 months).

Previous studies have reported on the efficacy of unilateral STN DBS for patients with prominently asymmetrical

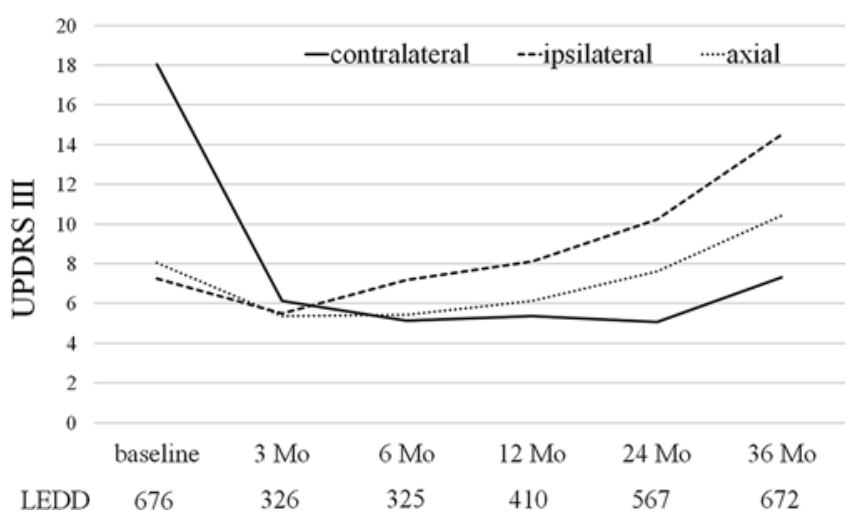

FIG. 1. Serial changes in mean UPDRS III subscores of all patients for 3 years after surgery. Following this, patients were not evaluated using the same schedule; therefore, mean regular timeline data were not available (see text for more details). The LEDD values are in milligrams. contralateral = contralateral to the side of the first unilateral surgery; ipsilateral = ipsilateral to the side of the first unilateral surgery.

parkinsonism, and authors have suggested that baseline asymmetry is an important factor when choosing between unilateral and bilateral surgery. $3,7,8,11,14$ A previous study by Taba et al. reported that 15 of 21 patients (71\%) had a mean interval between the first and second surgery of 3.5 years. ${ }^{14}$ In the present study, 7 patients $(87.5 \%)$ were potential or actual candidates for a second surgery. The patients included in our study had more prominently asymmetrical PD at baseline than those in previous studies; therefore, we argue that baseline asymmetry is not sufficient to indicate initial unilateral STN DBS as a long-term treatment strategy. As stated in previous studies, simultaneous bilateral surgery at a younger age is preferable to 2 unilateral surgeries at different time points because 1) older age is related to a higher risk of surgical complications and reduced improvements, 2) 2-stage surgery typically confers a greater financial burden, and 3) repetitive pre- and postoperative procedures can cause additional stress to the patients. . $^{5,9,12,16}$

Patient 8 was the only patient who remained satisfied with the unilateral STN DBS throughout the follow-up period. Interestingly, this patient's baseline asymmetry was less prominent than that of the other patients (ratio of UPDRS III score between both sides was 2.14 for patient 8 vs a mean of 3.59 for the other patients). Patient 8 did not experience side-reversal of the more affected side during the first 3 years after the initial surgery; in contrast, the other patients experienced side-reversal at a mean of 9.4 months (range 3-36 months) after the initial surgery in the off-medication and on-stimulation state. The postural instability/gait disturbance (PIGD) score at baseline for patient 8 was 0 , compared with a mean score of 1.8 (range $0.5-3.5)$ for the other patients. In addition, patient 8 was older than the mean age of the other patients at the time of the first surgery (67 vs mean 57 years), and his baseline off-medication UPDRS III score was lower than the mean score of the other patients (26.5 vs 33.4). There was no difference in the ratio of the UPDRS III score related to tremor vs nontremor symptoms (rigidity, bradykinesia, and PIGD).

This study has several limitations. First, it is a retrospective observational study. In addition, we followed a small 


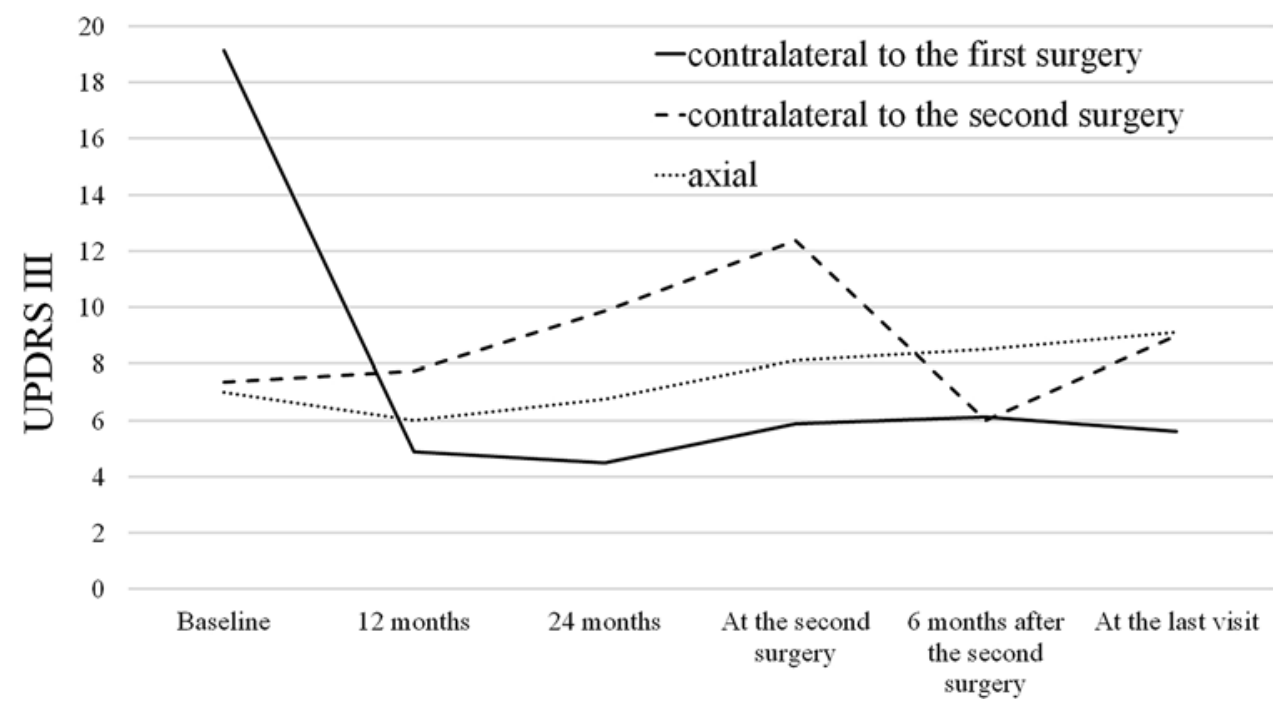

FIG. 2. Data from the 4 patients who had a second surgery. The mean time to the final follow-up evaluation was 45 months (range 33-59 months) after the second surgery.

number of patients; therefore, our conclusion that initial bilateral STN DBS should be considered even in patients with highly asymmetrical PD requires further confirmation in a larger cohort. Nevertheless, the fact that $50 \%$ of the patients (potentially 87.5\%) underwent a second surgery 5 years after the initial unilateral surgery favors our conclusion. The patients included in this study had more prominent baseline asymmetry than those in previous studies, and although the number of patients in the study group was small, they could be regarded as representative of patients with asymmetrical PD. Only 1 patient declined a second surgery during follow-up; therefore, statistical comparisons of the clinical factors that affect the decision for a second surgery were not possible. A further limitation is the lack of nonmotor symptom analysis in the patients, because the decision to undergo a second surgery can be based on nonmotor, in addition to motor, symptoms.

\section{Conclusions}

This study provides further evidence that, as a longterm treatment strategy, simultaneous bilateral STN DBS should be considered for patients with PD even if they have prominently asymmetrical motor symptoms.

\section{Acknowledgments}

This study was supported in part by a grant from the Korea Health 21 R\&D Project, Ministry of Health and Welfare, Republic of Korea (AO300001); from the Korea Institute of Planning \& Evaluation for Technology in Food, Agriculture, Forestry, and Fisheries, Republic of Korea (311011055SB0200); from the Korea Healthcare Technology R\&D Project, Ministry of Health \& Welfare, Republic of Korea (HI11C21100200); from the Industrial Strategic Technology Development Program (10050154); from the Bio \& Medical Technology Development Program of the NRF funded by the Korean government, MSIP(2015M3C7A1028926); and from the Cooperative Research Program of Basic Medical Science and Clinical Science, Seoul National University College of Medicine (800-20160093). We deeply appreciate a generous donation from Mr. Chung Suk-Gyoo and the Shinyang Cultural Foundation.

\section{References}

1. Alberts JL, Hass CJ, Vitek JL, Okun MS: Are two leads always better than one: an emerging case for unilateral subthalamic deep brain stimulation in Parkinson's disease. Exp Neurol 214:1-5, 2008

2. Bastian AJ, Kelly VE, Revilla FJ, Perlmutter JS, Mink JW: Different effects of unilateral versus bilateral subthalamic nucleus stimulation on walking and reaching in Parkinson's disease. Mov Disord 18:1000-1007, 2003

3. Chung SJ, Jeon SR, Kim SR, Sung YH, Lee MC: Bilateral effects of unilateral subthalamic nucleus deep brain stimulation in advanced Parkinson's disease. Eur Neurol 56:127132,2006

4. Kim HJ, Paek SH, Kim JY, Lee JY, Lim YH, Kim DG, et al: Two-year follow-up on the effect of unilateral subthalamic deep brain stimulation in highly asymmetric Parkinson's disease. Mov Disord 24:329-335, 2009

5. Kumar R, Lozano AM, Kim YJ, Hutchison WD, Sime E, Halket E, et al: Double-blind evaluation of subthalamic nucleus deep brain stimulation in advanced Parkinson's disease. Neurology 51:850-855, 1998

6. Kumar R, Lozano AM, Sime E, Halket E, Lang AE: Comparative effects of unilateral and bilateral subthalamic nucleus deep brain stimulation. Neurology 53:561-566, 1999

7. Linazasoro G, Van Blercom N, Lasa A: Unilateral subthalamic deep brain stimulation in advanced Parkinson's disease. Mov Disord 18:713-716, 2003

8. Nakamura K, Christine CW, Starr PA, Marks WJ Jr: Effects of unilateral subthalamic and pallidal deep brain stimulation on fine motor functions in Parkinson's disease. Mov Disord 22:619-626, 2007

9. Papapetropoulos S, Salcedo AG, Singer C, Gallo BV, Jagid JR: Staged unilateral or bilateral STN-DBS? Mov Disord 23:775, 2008

10. Park K, Lim YH, Jang M, Kim A, Kim HJ, Paek SH, et al: Battery life matters in deep brain stimulation. Stereotact Funct Neurosurg 96:65-66, 2018

11. Samii A, Kelly VE, Slimp JC, Shumway-Cook A, Goodkin R: Staged unilateral versus bilateral subthalamic nucleus stimulator implantation in Parkinson disease. Mov Disord 22:1476-1481, 2007

12. Schüpbach WM, Maltête D, Houeto JL, du Montcel ST, Mallet L, Welter ML, et al: Neurosurgery at an earlier stage of 
Parkinson disease: a randomized, controlled trial. Neurology 68:267-271, 2007

13. Slowinski JL, Putzke JD, Uitti RJ, Lucas JA, Turk MF, Kall BA, et al: Unilateral deep brain stimulation of the subthalamic nucleus for Parkinson disease. J Neurosurg 106:626-632, 2007

14. Taba HA, Wu SS, Foote KD, Hass CJ, Fernandez HH, Malaty IA, et al: A closer look at unilateral versus bilateral deep brain stimulation: results of the National Institutes of Health COMPARE cohort. J Neurosurg 113:1224-1229, 2010

15. Walker HC, Watts RL, Guthrie S, Wang D, Guthrie BL: Bilateral effects of unilateral subthalamic deep brain stimulation on Parkinson's disease at 1 year. Neurosurgery 65:302310, 2009

16. Welter ML, Houeto JL, Tezenas du Montcel S, Mesnage V, Bonnet AM, Pillon B, et al: Clinical predictive factors of subthalamic stimulation in Parkinson's disease. Brain 125:575-583, 2002

\section{Disclosures}

B.J. reports research grants from the Ministry of Health and Welfare, Seoul National University Hospital, Sinyang Cultural Foundation, Korean Movement Disorder Society, Boryung Pharm., Novartis Korea, Ipsen Korea, Samil Pharmaceuticals, Abbvie Korea, UCB Korea, Lundbeck Korea, and Sandoz Korea; travel grants from the Korea Research-Based Pharmaceutical Industry Association, Korean Pharmaceutical Manufacturers Association, Seoul National University, and Seoul National University Hospital. Han-Joon Kim reports a research grant from Seoul National University Hospital and the Ministry of Education, Republic of Korea. S.H.P. reports research grants from the Korea Institute of
Planning \& Evaluation for Technology in Food, Agriculture, Forestry, and Fisheries; Korea Healthcare Technology R\&D Project; Ministry of Health \& Welfare; Industrial Strategic Technology Development Program 10050154; Bio \& Medical Technology Development Program of the NRF funded by the Korean government; MSIP; and Basic Medical Science and Clinical Science, Seoul National University College of Medicine (800-20160093).

\section{Author Contributions}

Conception and design: Jeon, Ehm, Han-Joon Kim, Paek. Acquisition of data: Ehm, JY Kim, Lee, Yun, YE Kim, Yang, Lim, Hee Jin Kim. Analysis and interpretation of data: Jeon, Ehm, Han-Joon Kim. Drafting the article: Ehm. Critically revising the article: all authors. Reviewed submitted version of manuscript: Jeon, Han-Joon Kim, JY Kim, Lee, Yun, YE Kim, Yang, Lim, Hee Jin Kim, Paek. Approved the final version of the manuscript on behalf of all authors: Jeon. Statistical analysis: Ehm. Administrative/technical/material support: Lim. Study supervision: Jeon, Han-Joon Kim, Paek.

\section{Supplemental Information}

\section{Previous Presentations}

The results of this study were presented in poster form at the International Congress of Parkinson's Disease and Movement Disorders, Berlin, Germany, June 20, 2016.

\section{Correspondence}

Beomseok Jeon: Seoul National University, Seoul, Korea. brain@ snu.ac.kr. 